\title{
Voltage Breakdown Limits at a High Material Temperature for Rapid Pulse Heating in a Vacuum
}

\author{
P.A. Pincosy \\ R. Speer
}

This paper was prepared for submittal to the

Institute of Electrical and Electronics Engineers

12th International Pulsed Power Conference

Monterey, $\mathrm{CA}$

June 27-30, 1999

June 7, 1999

This is a preprint of a paper intended for publication in a journal or proceedings.

Since changes may be made before publication, this preprint is made available with the understanding that it will not be cited or reproduced without the permission of the author. 


\section{DISCLAIMER}

This document was prepared as an account of work sponsored by an agency of the United States Government. Neither the United States Government nor the University of California nor any of their employees, makes any warranty, express or implied, or assumes any legal liability or responsibility for the accuracy, completeness, or usefulness of any information, apparatus, product, or process

disclosed, or represents that its use would not infringe privately owned rights. Reference herein to any specific commercial product, process, or service by trade name, trademark, manufacturer, or otherwise, does not necessarily constitute or imply its endorsement, recommendation, or favoring by the United States Government or the University of California. The views and opinions of authors expressed herein do not necessarily state or reflect those of the United States Government or the University of California, and shall not be used for advertising or product endorsement purposes. 


\title{
VOLTAGE BREAKDOWN LIMITS AT A HIGH MATERIAL TEMPERATURE FOR RAPID PULSE HEATING IN A VACUUM*
}

\author{
P. A. Pincosy and R. Speer \\ Lawrence Livermore National Laboratory \\ Livermore CA. 94550
}

\begin{abstract}
The proposed Advanced Hydro Facility (AHF) is required to produce multi-pulse radiographs. Electron beam pulse machines with sub-microsecond repetition are not yet available to test the problem of electron beam propagation through the hydro-dynamically expanding plasma from the nearby previously heated target material. A proposed test scenario includes an ohmically heated small volume of target material simulating the electron beam heating, along with an actual electron beam pulse impinging on nearby target material. A pulse power heating circuit was tested to evaluate the limits of pulse heating a small volume of material to tens of kilo-joules per gram. The main pulse heating time (50 to $100 \mathrm{ns)} \mathrm{was}$ to simulate the electron beam heating of a converter target material. To avoid skin heating non-uniformity a longer time scale pulse of a few microseconds first heats the target material to a few thousand degrees near the liquid to vapor transition. Under this state the maximum electric field that the current carrying conductor can support is the important parameter for insuring that the $100 \mathrm{~ns}$ heating pulse can deposit sufficient power. A small pulse power system was built for tests of this limit. Under cold conditions the vacuum electric field hold-off limit has been quoted as high as many tens of kilovolts per centimeter. The tests for these experiments found that the vacuum electric field hold-off was limited to a few kilovolts per centimeter when the material approached melting temperatures. Therefore the proposed test scenario for AHF was not achievable."
\end{abstract}

\section{INTRODUCTION}

The proposed Advanced Hydro Facility (AHF) is required to produce multi-pulse radiographs using high power electron beam conversion. There are no multi-pulse high power machines to appropriately test the problem of electron beam propagation through the hydro-dynamically expanding plasma from a previous pulse. One method produces a blow-off plasma through the application of laser power to the surface. This expanding plasma ${ }^{1}$

\footnotetext{
* Work performed under the auspices of the U. S. Department of Energy by the Lawrence Livermore National Laboratory under contract No. W-7405-ENG-48
}

impacts the propagation of the electron beam to the converter target material. The difficulty is that the plasma characteristics are quite different in terms of temperature and thus expansion speed ( several times larger). Another possibility proposed here is to produce an ohmically heated small volume of material. The bulk heating is similar to beam energy deposition.

We evaluate the significant parameters involved in depositing the energy into the small volume of material and explore their scaling at high energy densities where short pulse power heating times may allow for only partial vaporization. We propose a pulse power design to enable relatively uniform pulse power deposition on the short time scale of 50 to $100 \mathrm{~ns}$ and yet allow current penetration into the material which at room temperature has a skin depth much smaller than the thickness. To drive the high power deposition, the conductor must support a high electric field. Such a field may be too high to be supported on the material surface, especially in a vacuum. There are indications ${ }^{2}$ that a sufficiently high electric field $(\sim 50 \mathrm{kV} / \mathrm{cm})$ can be maintained at room temperature. A 2D hydrodynamics code $\left(\mathrm{CALE}^{3}\right)$ capable of including the MHD aspects was used in a co-axial approximating geometry to study the current penetration and heating uniformity. We explored the electric field hold-off issue with a small-scale experiment in a vacuum which can match the required electric field under similar dynamical conditions of the material thermal state. One issue was to measure the average material resistivity during the high power deposition when the hydrodynamic time scale is of the same order or longer than the heating time. The experimental results show the limit of electric field hold-off under the resultant thermal and dynamical conditions and the resistivity equation of state is compared to other results.

\section{SCALING FOR OHMIC HEATING}

The electron beam from an induction linac accelerator typically deposits power during 40 to $60 \mathrm{~ns}$ into a material converter of $0.1 \mathrm{~cm}$ thickness. The beam spot size can be as small as one millimeter in diameter. The peak power $P_{b}$ into this spot for the AHF beam is $120 \mathrm{GW}$, of which $14 \mathrm{GW}$ is deposited into the material. We match the ohmic heating power into the same volume with a current conduction path of $l$, and a voltage drop of $V$. Thus the 
electric field along the conductor is $E=\sqrt{\eta P_{b}}$. Assuming cross-section dimensions of width $w$ and thickness of $\delta$, the required pulse power supply current

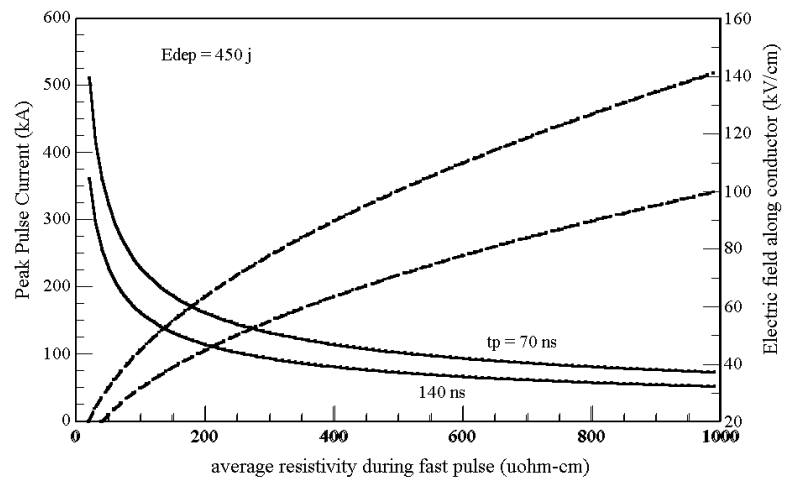

Figure 1. Required peak pulse current ond resulting electric field to achieve 450 joule energy deposition or $25 \mathrm{kj} / \mathrm{gm}$ energy density.

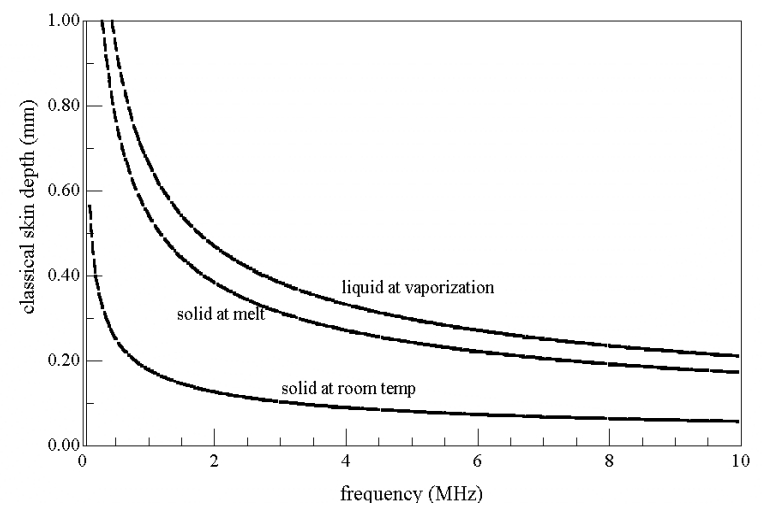

Figure 2. Skin thickness dependance on material temperature and frequency

$I=t w \sqrt{P_{b} / \eta}$. The effect of skin depth can be roughly related to the heating time scale $\tau_{p}$, or $\omega=\pi / \tau_{p}$. These parameters are presented in Figure 1 showing the required peak pulse current and electric field as a function of the resistivity $\eta$ at roughly the peak power transfer. The parameter $\omega$ represents the rate at which the power is delivered. It is immediately obvious that the required current and electric field must be very high and any increase in material resistiviy would reduce the current and increase the electric field. For a converter material of tantalum the classical skin depth is plotted (Fig 2) as a function of frequency for three temperatures of interest.
At room temperature and for a one millimeter thick material, the skin depth will not allow a heating frequency greater than one $\mathrm{MHz}$ up to about $2000 \mathrm{C}$, but at liquid temperatures the skin depth will allow up to a few $\mathrm{MHz}$ heating rate. Typical electric field hold-off in air is 5 to 10 $\mathrm{kV} / \mathrm{cm}$, depending on the duration. In a good vacuum well below the knee of the Paschen minimum the electric field hold-off may be better or worse depending on the material temperature and evaporation-to-ionization state. Vitkovitsky and Scherrer ${ }^{4}$ measured electric field holdoff across opening fuses as high as $20 \mathrm{kV} / \mathrm{cm}$ in a $10^{-5}$ torr vacuum for pulses less than one microsecond. For nanosecond pulses, fields ${ }^{5}$ as high as $150 \mathrm{kV} / \mathrm{cm}$ were obtained across opening fuses.

\section{CO-AXIAL MHD MODELING}

The typical electron beam converter is a slab of tantalum one millimeter thick. The electron beam energy deposition during the heating pulse is modeled assuming the electrons follow straight-line trajectories and deposit power according to the radiation mean free path. A beam gaussian power profile is assumed for the hydrodynamic response calculation. For the typical FXR parameters of $18 \mathrm{MeV}, 2.8 \mathrm{kA}$ and $60 \mathrm{~ns}$ into a beam spot of $1.8 \mathrm{~mm}$ (fwhm), the end of pulse material energy is about 10 $\mathrm{kj} / \mathrm{gm}$. For the proposed AHF facility the equivalent energy density would be $50 \mathrm{kj} / \mathrm{gm}$. To match these

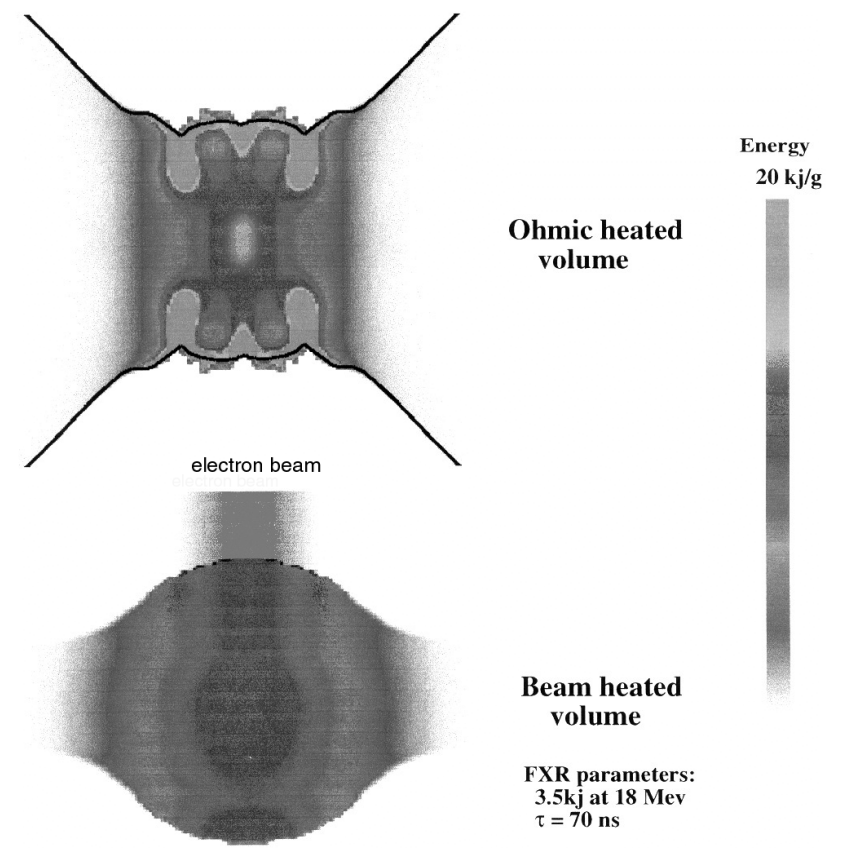

Figure 3. Calculated energy content of a beam-heated tantalum target compared to an ohmically-heated equivalent volume. 
conditions would be much more difficult. The lower energy density will however provide an expanding plasma through which useful beam propagation studies may be made.

We model this heated volume with CALE using its MHD capability. Since the code handles 2D coaxial geometry we approximate the energy deposition by a onemillimeter long tantalum conductor fed by a conical divergent electrode co-axial to a return conductor. The heating current $(125 \mathrm{kA})$ pulse first consists of a 5 microsecond pulse which raises the material temperature for increased resistivity and larger skin depth. A $100 \mathrm{~ns}$ second pulse at much higher peak current (1.2 MA) is applied to deposit sufficient energy to partially match the beam heated material energy. In Fig 3 the calculated energy distribution is compared. The material energy in the core of the tantalum is $10 \mathrm{kj} / \mathrm{gm}$ but the skin current flow down the conical electrode has created a higher energy zone $(\sim 20 \mathrm{kj} / \mathrm{gm})$ on the surface which pinches inward as lobes toward the axis. This region has expanded in density and has a higher calculated resistivity. The expanding material would then have a higher sound speed and the expansion would be more rapid than the beam heated material. The energy density is about twice, so that the expanding material speed will be about $\sqrt{2} c_{b}$ of the beam-produced expansion. The calculated radial velocity at the end of the heating pulse is $0.3 \mathrm{~cm} / \mathrm{us}$. There may well be some much hotter plasma on the surface produced by the high electric fields supported along this conductor section. If not, the ohmic heating will produce an expanding-plasma not too grossly different than the beam-heated plasma. The code uses a four-phase resistivity model and indicates a liquid-like resistivity persisting through the main heating pulse. Since this would probably be the case for any pulse power application, a significantly higher pulse power supply current is required than would be required if the material had time to expand into the vapor-state during the heating pulse.

\section{EXPERIMENTAL CIRCUIT SETUP}

A circuit design capable of achieving a preheat pulse before the main energy deposition can be achieved using two parallel capacitor supply circuits each separately switched. The fast circuit can be inductively isolated from the slow circuit since only a half wave pulse from each is desired. The second pulse is applied at the current zero crossing of the first which can be crow-barred at the end of the fast pulse application. If the second pulse has a reasonable impedance match to the load at peak resistance little energy remains for ringing. Another method is to match the supply into a static load (resistor value for critical damped pulse) which is larger than the target load. This design will help to avoid energy cycling beyond the first pulse. However this design does not permit a very efficient power transfer and makes a short pulse more difficult to achieve. Low impedance transmission lines with sufficient energy storage are within practical range for this application and may be made to match the load for the main period of power transfer.

For our small-scale experiment we chose to operate the fast circuit capacitor into a matched resistor which could be changed for experimental flexibility. The circuit shown in Fig 4 was setup with the fast line at ground potential. The slow heater circuit was inductively isolated on either side of the capacitor. Both capacitors were switched by spark gaps. As modeled by PSPICE the half cycle time for the slow circuit was 5 us (Fig 4) and the fast drive into $1.6 \mathrm{ohms}$ had a half cycle time of under 100ns. For this modeling the tantalum resistance equation of state up to vaporization was used from Gallob et al $^{6}$ and Shaner et $\mathrm{al}^{7}$.

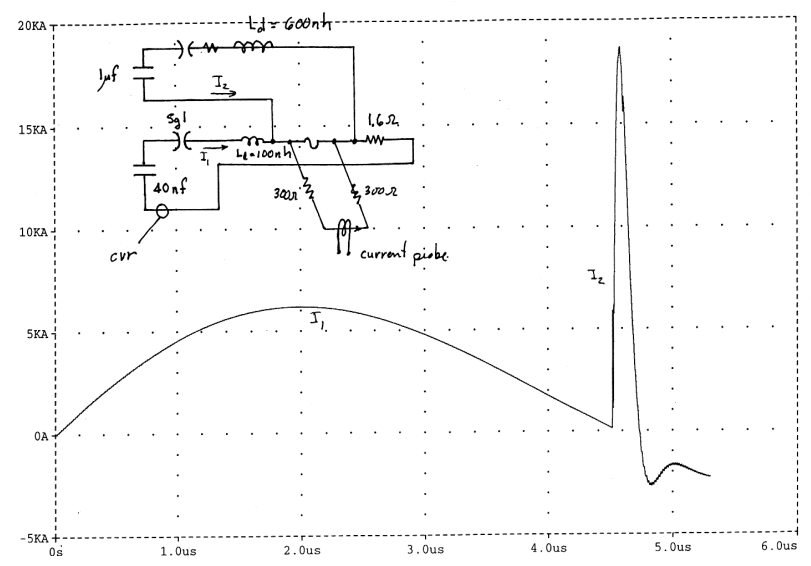

Figure 4. Calculated current through the fuse for the inset circuit: circuit element values are those for the small sacle experiment

Beyond the liquid state we fabricated an equation of state scaled similar to an equation of state measured using a bursting foil of copper ${ }^{8}$. A priori we knew that the equation of state would be very dependent on the time scale and foil size. The experiment fast line, being required to function in a vacuum, was made as a kapton layered wide line. A six inch diameter glass cylinder was vacuum sealed onto the kapton and supported below by a flange. A base pressure of $10^{-6}$ torr was obtained in the vacuum-chamber by a turbo pump plus a liquid-nitrogencooled coil inserted into the chamber. The short tantalum fuse is clamped across the exposed gap in the top conducting strip. The voltage $V_{b}$ across the fuse is measured using the conducting strip going to the side outside the vacuum chamber. For diagnostics we measured the current in the slow bank with a current 
transformer and a current viewing resistor at the capacitor in the fast bank. The voltage across the fuse was measured using a current transformer to monitor the current through a calibrated $620 \mathrm{ohm}$ shunt resistor. Also we measured the voltage across the matched load resistance.

\section{EQUATION OF STATE DATA}

From the measured fuse current and voltage drop, which includes the inductive drop, we assume an inductance sufficient to give zero voltage drop at the beginning of the fast pulse current rise. This resistive component of the fuse voltage divided by the measured circuit current provides a time dependent fuse resistance. The integral of the power deposited in the resistance provides the energy density and we plot the resistivity

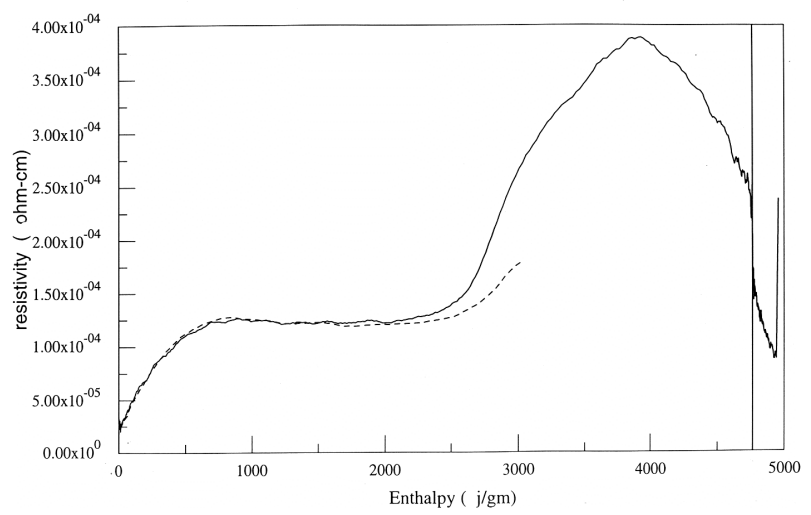

Figure 5. Tantalum resistivity equation of state into the vapor state with the slow (5us) time.

equation of state assuming the fuse was uniformly heated over its length. First we used a longer fuse to reduce the effect of clamp and end variation on the resistance. Also only the slow bank circuit was activated to allow time for the liquid to vapor transition. We observed the transition (Fig 5) from the liquid to the vapor state and the overall increase in resistivity is over 100 times the room

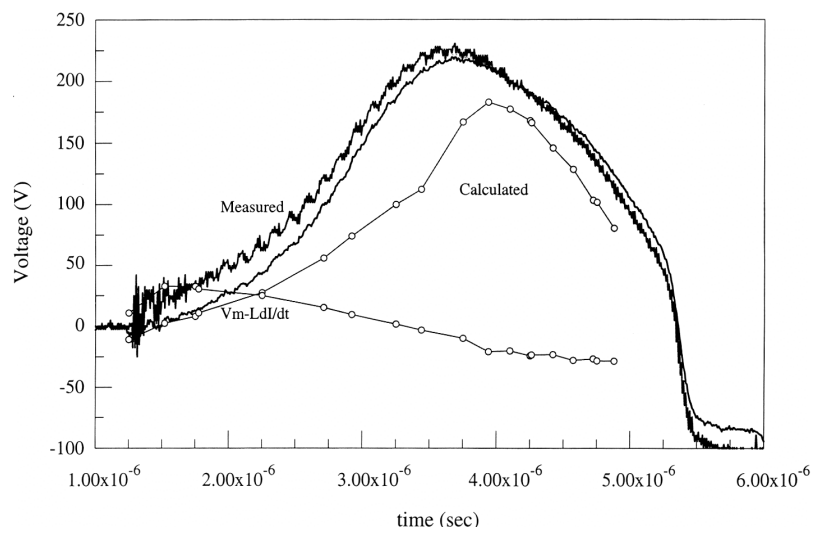

Figure 6. Comparison of the measured and calculated fuse voltage from MHD heating.

temperature value. Many fuse opening experiments use some form of tamped copper foil which, depending on the power deposition rate and the tamping, give an equation of state ${ }^{8}$ similar to the curve shown.

For the short $(1 \mathrm{~mm})$ fuse the measured and calculated voltage for an equivalent cross-section and pulse power drive are compared in Fig 6. Apparently the relative geometry difference between the experimental foil and the axis-symmetric wire plays a significant role especially considering the end effects. Also the foil will have nonuniform heating at the edges. The peak voltage is however not too different. The measurement (Fig 7) during the fast
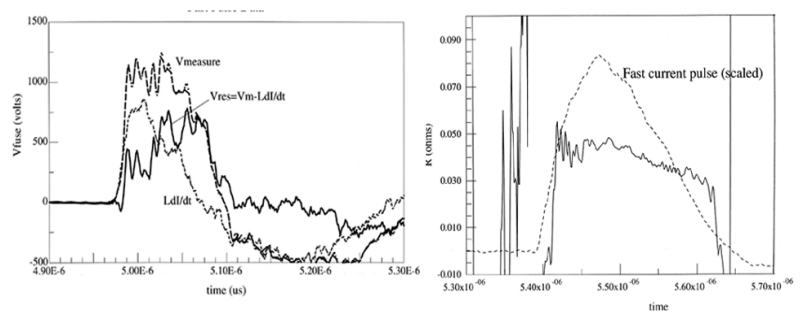

Figure 7. Typical fast pulse data and corresponding resistance.

portion of the pulse power was more difficult because of the non-matched power flow between the driver and the load. Also the line itself has some storage capacitance. During the fast pulse the material resistivity remains about the same as the liquid state resistivity. The energy deposition was less than $1 \mathrm{kj} / \mathrm{gm}$ during this pulse and was not as much as expected if vaporization had occurred which would produce a higher fuse resistance and a better pulse power transfer. In order to achieve the required energy per gram of a few tens of $\mathrm{kj} / \mathrm{gm}$ we would need to significantly increase the fast pulse current.

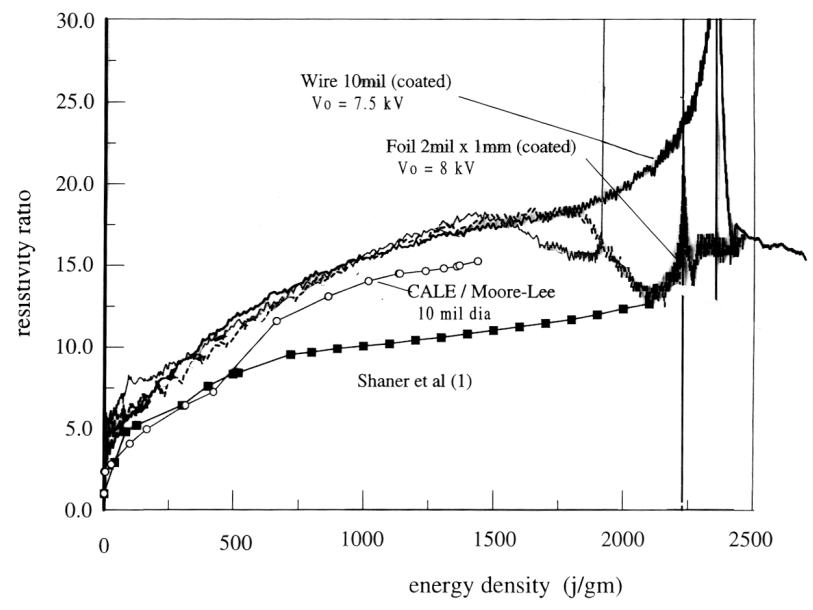


In Fig 8 we compare the measured equation of state with the more careful measurement by Shaner et. al where the end effects have been removed from the measurement. Our results are significantly higher perhaps because of end effects and the calculation seems to confirm this. Our measurement became noisy and inaccurate at the end of the slow pulse because the current is approaching zero.

\section{VOLTAGE BREAKDOWN}

The main goals for the small scale experiment were to measure the equation of state up to and during the fast power deposition, and to discover the breakdown voltage limits under operating conditions. Under atmospheric pressure the voltage across the fuse did not collapse during the heating pulses. Although we thought the resistance might increase significantly during the fast pulse as indicated by the equation of state shown in Fig 5 for the slow heating, it did not (Fig 8). We pumped the region down to below $10^{-5}$ torr to reduce the possibility of paschen breakdown. Under these conditions the $1.2 \mathrm{~mm}$ gap between the clamps held off $10 \mathrm{kV}$ under pulse conditions with no fuse across the gap.

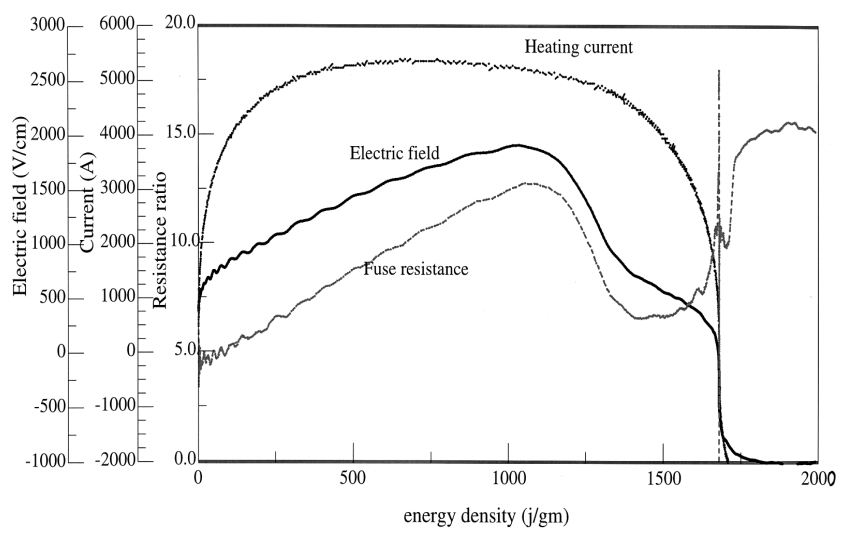

Figure 9. Fuse electric field fell during the slow heating as the material was in the liquid state.

When we applied the slow pulse to the fuse, the voltage across the gap increased with time (Fig 9) as expected but collapsed at an electric field between 2 and $2.5 \mathrm{kV} / \mathrm{cm}$. This resistance decrease varied, depending upon the rate of power deposition at the breakdown level. After current reversal the resistance appeared to return to the appropriate value but the electric field is now at 1 $\mathrm{kV} / \mathrm{cm}$. This impedance drop may be due to field ionization of the surface vapor.

Our conclusion was that for the high heating rate and thick conductors we could not expect the short fuse too support the electric field necessary. For much lower final energy levels this technique may be applicable.

\section{REFERENCES}

[1] S Sampayan, G. Caporaso, Y-J Chen, M. Garcia, T. Houck, R. Richardson, J. Weir, G. Westenskow, C. Crist, "Experimental investigation of beam optics issues at the bremsstrahlung converters for radiographic applications", UCRL-JC-130417, $19^{\text {th }}$ Int. LINAC Conf., (1998)

[2] M. Lehr, R. Korzekwa, H. Krompholz, and M. Kristiansen, "Increasing surface flashover potential using magnetic insulation", Proc. $7^{\text {th }}$ IEEE Pulse Power Conf., p812, (1989)

[3] R.E. Tipton, "A 2D Lagrange MHD Code" Megagauss Tech. and Pulsed Power Applications, Plenum Press, p299, (1987)

[4] I,M. Vitkovitsky and V.E. Sherrer, "Recovery characteristics of exploding wire fuses in air and vacuum" J. Appl. Phys. 52, p3012 (1981)

[5] N.E. Molau, J.E. Vernazza, R.A. Anderson, D.A. Goerz, and R.R. Heritsch, "Experimental investigation of a fast-acting exploding metallic foil opening switch", Proc. $7^{\text {th }}$ IEEE Pulse Power Conf., p975, (1989)

[6] R.Gallob, H. Jager, G. Pottlacher, "Recent results on thermophysical data of liquid niobium and tantalum", High Temperature-High Pressures, p207, 17, (1985)

[7] J.W. Shaner, G.R. Gathers, C. Minichino, "Thermophysical properties of liquid tantalum and molybdenum”, High Temperature-High Pressures, p331, 9, (1977)

[8] P. Poulsen, J.L. Cutting, R.S. Hawke, A.J. McAlice, P.A. Pincosy, J.B. Chase, "Inductive store opening switch and tests for energy transfer to a load", Megagauss Fields and Pulsed Power Systems, Nova Science, p579,(1990) 